\title{
Preparation and Characterization of PEGylated C18 Fatty Acids/Anti-SNAP25 Antibody-Targeted Liposomes
}

\author{
Lai Ti Gew ${ }^{*}{ }^{1,2}$, Vicit Rizal Eh $\operatorname{Suk}^{2}$ and Misni Misran ${ }^{3}$
}

${ }^{1}$ Department of Biological Sciences, School of Science and Technology, Sunway University, No. 5, Jalan Universiti, Bandar Sunway, 47500 Subang Jaya, Selangor, Malaysia; ${ }^{2}$ Department of Chemistry, Faculty of Science, University of Malaya, 50603 Kuala Lumpur, Malaysia; ${ }^{3}$ Department of Chemistry, Faculty of Science, University of Malaya, 50603 Kuala Lumpur, Malaysia

\begin{abstract}
Background: Unsaturated C18 fatty acids, such as oleic acid (L1), linoleic acid (L2), and linolenic acid (L3), are a good choice of lipids to prepare liposomes. They are inexpensive, biocompatible, nontoxic, and readily available compared with phospholipids. Moreover, cis-double bonds of unsaturated fatty acids prevent the packing of molecules which increases membrane fluidity in liposomes making them a good choice of starting materials to prepare liposomes.
\end{abstract}

Objective: Unsaturated C18 fatty acid liposomes, as well as their PEGylated and nonPEGylated antibody-targeted liposomes, were prepared and characterized.

A R T I C L E H IS T O R Y

Received: May 14, 2018

Revised: August 08, 2018

Accepted: September 06, 2018

DOI:

10.2174/2212796812666180912113156
Method: The particle size and zeta potential of the prepared liposomes $(1 \mathrm{mM}, \mathrm{pH}=7.4)$ for 28 and 14 days, respectively, were monitored and characterized. Membrane-bound antibodies Anti-SNAP25 (AS25) and DOPE PEG2000 (DP) were conjugated to pure C18 fatty acid liposomes to achieve stable fatty acid formulations.

Results: The mean particle sizes of pure L1, L2, and L3 liposome solutions were 125, 129, and $122 \mathrm{~nm}$ respectively, while their polydispersity index values were $0.28,0.21$, and 0.40 respectively. A large negative zeta potential value of $45 \mathrm{mV}$ was observed due to anionic carboxylate head-group of pure liposomes. The incorporation of AS25 into L1/DP, L2/DP, and L3/DP liposome solutions stabilized their mean particle size and zeta potential measurements over 28 and 14 days, respectively.

Conclusion: L1/DP/AS25 was found to be the most stable PEGylated antibody-targeted liposome system because its particle size remained between 90 and $125 \mathrm{~nm}$ in 28 days. Transmission electron microscopy observations also supported the incorporation of AS25 and DP on the membrane surface as predicted.

Keywords: Stealth liposomes, drug delivery systems, therapeutic proteins, biotechnology, nanotechnology, Liposomes.

\section{INTRODUCTION}

Proteins and peptides are important bioactive macromolecules that offer numerous advantages of highly specific and effective therapeutic

\footnotetext{
*Address correspondence to this author at the Department of Biological Sciences, School of Science and Technology, Sunway University, No. 5, Jalan Universiti, Bandar Sunway, 47500 Petaling Jaya, Selangor, Malaysia; Tel: +603-74918622; E-mail: janeg@sunway.edu.my
}

mechanisms of actions. Peptides that are highly specific small biomolecules act as substrates, inhibitors, or regulators and play important roles in monitoring biological functions [1]. For example, insulin as a peptide hormone is one of the most widely used drugs for diabetes. Due to their physiochemical properties, peptides have limited ability to cross cell membranes to reach intracellular target sites. Most peptides, thus, need to be entrapped into nanoscale delivery systems to effi- 
ciently facilitate their intracellular delivery. Protein therapeutics are also limited by several pharmaceutical issues, such as high molecular weight, and in vitro and in vivo short half-lives. These issues cause side effects, such as immunogenicity, resulting from repeatable injections of rapidly degradable protein drugs over a long diagnostic period. In addition, there is a lack of an effective way to deliver functional proteins across plasma membranes due to their large molecular size and biochemical properties [2-4]. Therapeutic proteins, such as antibodies, can be conjugated onto the surface of liposomes. Besides, nucleic acids (DNA/RNA), virus antigens, and therapeutic enzymes can be encapsulated into liposomes.

Antibody-targeted liposomes are made of conjugating antibodies to the lipid bilayer of a liposome surface, which promotes a specific interaction with cancer cells [5-8]. Pharmacokinetic analysis and therapeutic studies reveal that antibody-targeted liposomes have considerable potential to be used as a drug delivery system for cancer therapy. Such a system optimizes the drug delivery to tumor cells, reduces the exposure of highly toxic anticancer drug to healthy cells, and minimizes side effects [9-12].

Unsaturated C18 fatty acids are a good choice of lipids to prepare liposomes $[13,14]$ as they are inexpensive, biocompatible, nontoxic, and readily available. Moreover, the cis-double bond(s) of unsaturated fatty acids make them the good choice of starting material to prepare liposomes. The presence of a cis-double bond prevents the packing of molecules, which increases the membrane fluidity in liposomes. Membrane fluidity allows drug en- capsulation and conjugation of the antibody. We have reported the preparation of stealth and nonstealth fatty acid liposomes and their capability in drugs encapsulation [15-17]. The most notable drawback of fatty acid liposomes is their physical and chemical instabilities during storage. In fact, this is a general drawback for most of liposomal formulations, including some phospholipid liposomes. Therefore, membrane-bound antibodies and poly(ethylene glycol) can be conjugated to pure C18 fatty acid liposome solutions (as illustrated in Fig. 1) to stabilize fatty acid formulations, because lipid-protein interactions naturally occur in biological membranes.

In the present work, we prepared liposomes of unsaturated $\mathrm{C} 18$ fatty acids with the incorporation of Anti-SNAP25 (AS25) and DOPE PEG2000 (DP). The particle size and zeta potential of the prepared liposomes for 28 and 14 days, respectively, were monitored and characterized. This research serves as the outcome of our previous studies on intermolecular interactions between AS25 (and DP) and C18 fatty acids using Langmuir technique $[18,19]$. Briefly, AS25 is a membranebound, presynaptic nerve terminal protein that plays an essential role in vesicle membrane fusion events with the plasma membrane. SNAP-25 is a soluble protein having a molecular weight of 25 $\mathrm{kDa}$, which contains 206 amino acids [20]. Membrane fusion is a vital process in biological systems and facilitates the transport of molecules across a membrane. It is crucial for the entry of encapsulated drugs into the targeted site [21]. Membrane fusion during intracellular transport is (a)

(c)

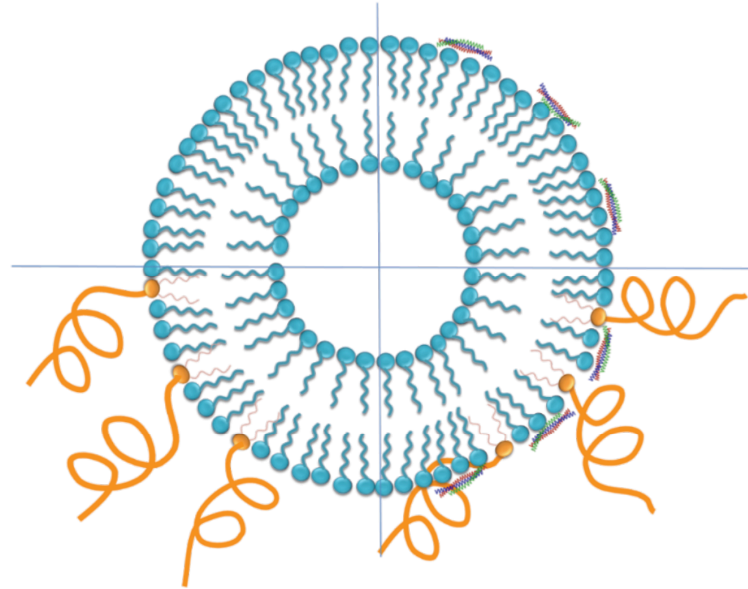

(b)

(d)

Fig. (1). A schematic diagram illustrates the surface of (a) liposomes, (b) antibody-targeted liposomes, (c) stealth liposomes, and (d) stealth antibody-targeted liposomes. 
thought to be mediated by a large family of proteins known as soluble $N$-ethylmaleimide-sensitive factor protein receptors (SNARE) proteins.

This study plays an important role in developing new antibody-targeted liposome formulations. This work serves as a useful reference to improve fatty acid liposome formulations that are currently overlooked.

\section{MATERIALS AND METHODS}

\subsection{Materials}

C18 fatty acids, such as oleic acid (cis-9octadecenoic acid, $\geq 99 \%$ ), linoleic acid (cis, cis9,12-octadecadienoic acid, $\geq 99 \%$ ), linolenic acid (cis, cis, cis-9,12,15-octadecatrienoic acid, $\geq$ 99\%), and AS25 (Product number S9684) were purchased from Sigma-Aldrich (Palo Alto, CA, USA). AS25 was supplied as an IgG fraction of antiserum (using rabbit as host species) in $0.01 \mathrm{M}$ phosphate-buffered saline (PBS) of $\mathrm{pH}=7.4$, containing $15 \mathrm{mM}$ sodium azide as a preservative. The concentration of AS25 was $9.55 \mu \mathrm{g} / \mu \mathrm{l}$. It was stored at $-20^{\circ} \mathrm{C}$ in a freezer. 1,2-dioleoyl-snglycero-3-phosphoethanolamide-N-

[methoxy(polyethyleneglycol)-2000] (ammonium salt), DOPE PEG2000 (DP) having molecular weight of 2801.47 amu, was purchased from Avanti Polar Lipids, USA. DP is a white powder that was kept at $-20^{\circ} \mathrm{C}$ in a freezer when not in use.

Analytical grade chloroform $\left(\mathrm{CHCl}_{3}\right)$ was purchased from Merck, USA and used to dissolve lipids. $\mathrm{CHCl}_{3}$ was double-distilled at its boiling point $\left(61.2^{\circ} \mathrm{C}\right)$ before use. PBS tablets were purchased from Sigma-Aldrich. One tablet of PBS that was completely dissolved in $200 \mathrm{ml}$ of distilled water yielded $0.01 \mathrm{M}$ PBS ( $\mathrm{pH} \mathrm{7.4)}$ at $25^{\circ} \mathrm{C}$. Phosphotungstic acid (PTA) hydrate for transmission electron microscopy (TEM) negative staining was purchased from Fluka and stored in a desiccator when not in use.

\subsection{Preparation of Unsaturated C18 Fatty Ac- ids/AS25, C18 Fatty Acids/DP, and C18 Fatty Acids/DP/AS25 Liposomes}

Oleic acid (L1) liposome solution of $1 \mathrm{mM}$ was prepared by mixing L1 in $1 \mathrm{ml}$ of $0.05 \mathrm{M} \mathrm{NaOH}$ aqueous solution, $\mathrm{pH}$ of the solution was adjusted to 7.4 using $0.05 \mathrm{M} \mathrm{HCl}$. Subsequently, the solu- tion was topped up using $0.01 \mathrm{M}$ PBS solution in a $5 \mathrm{ml}$ volumetric flask. The procedures for preparing stock solutions of linoleic acid (L2) and linolenic acid (L3) were the same. A desired volume of $\operatorname{AS} 25(10,25,50,75$, or $100 \mu 1)$ was added into $1 \mathrm{mM}$ of L1, L2, and L3 liposome solutions [18] using a Hamilton microsyringe that was precise to $0.5 \mu 1$. The mixtures were stirred overnight before characterizations.

C18 fatty acids/DP liposomes were prepared by lipid thin-film hydration method $[22,23]$. Briefly, various mole ratios of L1/DP, L2/DP, and L3/DP were mixed in $\mathrm{CHCl}_{3}$ in a round bottom flask. Thin films of lipids were created on the flask using a rotary vacuum evaporator to remove the residual organic solvent. The dry film was then hydrated using $0.01 \mathrm{M}$ PBS to swell the convolute into liposomes. The resulting solutions were adjusted to $\mathrm{pH} 7.4$ using $0.05 \mathrm{M} \mathrm{NaOH}$ or $\mathrm{HCl}$. Subsequently, sonication of the solution was performed to break multi-lamellar films into simple sheets of lamellar structures that eventually formed unilamellar liposomes. The suspension was then topped up with PBS solution. The mole ratio of C18 fatty acids/DP liposomes at 1 to 0.02 (or 50 to 1) was selected by referring to their stability assessment as shown in Fig. (2) and our previous LB findings [19]. The same volume of AS25 as above was added to individual vials containing $5 \mathrm{ml}$ of 1 $\mathrm{mM} \mathrm{C18}$ fatty acids/DP liposome solutions. The amount of AS25 that was added into C18 fatty acids and $\mathrm{C} 18$ fatty acids/DP liposome solutions were expressed in 3.6, 7.40, 16.8, and 25.2 nmole, as shown in Figs. (4-7). Both C18 fatty acids/DP liposomes and antibody-targeted $\mathrm{C} 18$ fatty acids/DP liposome solutions were stirred overnight before characterization.

\subsection{Measurement of Particle Size and Zeta Po- tential}

Mean particle size of liposomes, zeta potential, and Polydispersity Index (PDI) are commonly monitored parameters. The mean vesicle size and zeta potential were measured by dynamic light scattering (DLS) using Malvern Nano ZS (Malvern Instruments Ltd. UK). Using a disposable syringe, $1.0 \mathrm{ml}$ of liposomes was carefully transferred into $1 \mathrm{~cm}$ path length four-sided clear fluorescent quartz cuvette. The liposomes were analyzed using pre-set standard operation procedure for DLS to measure the average hydrodynamic 
size of liposomes and zeta potential measurements. A $4 \mathrm{~mW}$ He-Ne laser was employed to generate the laser beam that was detected by the backscattered detector at $173^{\circ}$ with respect to the scattering transmission beam. The scattered beam passed through the sample and then directed to compensation optic at $17^{\circ}$ from the scattering beam. The fluctuation in the frequency of the scattered beam was detected by the detector and its intensity was analyzed by the Malvern software. All measurements were performed in triplicate at $25^{\circ} \mathrm{C}$.

\subsection{TEM Imaging}

A drop of liposome solution was placed onto the high-quality formvar-coated copper grid (400 square mesh) and allowed to stand for $3 \mathrm{~min}$. The excess of solution was blotted with filtered paper, stained with $1 \%(\mathrm{w} / \mathrm{v})$ PTA for $1 \mathrm{~min}$, and allowed to air dry. The specimens were kept in a desiccator for 2 to 3 days and allowed to dry. Negative staining using PTA solution is an easy and widely used method for examining liposome structures at electron microscopy level. However, negative staining involves deposition of heavy atom stains (in this case, tungsten) because flattening of spherical or cylindrical liposome structures is commonly observed. PTA stained liposomes show bilayer moiety due to their strong affinity to PTA and become very electron-dense [24]. The images of liposomes were obtained by using Zeiss Libra ${ }^{\circledR}$ 120 TEM operated at an accelerating voltage of $120 \mathrm{kV}$.

\section{RESULTS AND DISCUSSION}

\subsection{Particle Size and Zeta Potential Analysis of C18 Fatty Acids and C18 Fatty Acids/DP Liposomes}

The mean particle size of pure L1, L2, and L3 liposomes solution ( $\mathrm{pH} 7.4)$ were 125,130 , and $120 \mathrm{~nm}$ respectively (Fig. 2). Their PDI values were $0.28,0.21$, and 0.40 respectively. Generally, PDI describes the uniformity degree of size distribution of particles. For a monodisperse sample, its PDI value is considered to be less than 0.1 . PDI of more than 0.5 suggests a broad distribution sample of mono-modal, reasonably narrow, and spherical samples. The stability assessment on pure liposome solutions demonstrated that the particle size was stable and remained less than $200 \mathrm{~nm}$ in a)

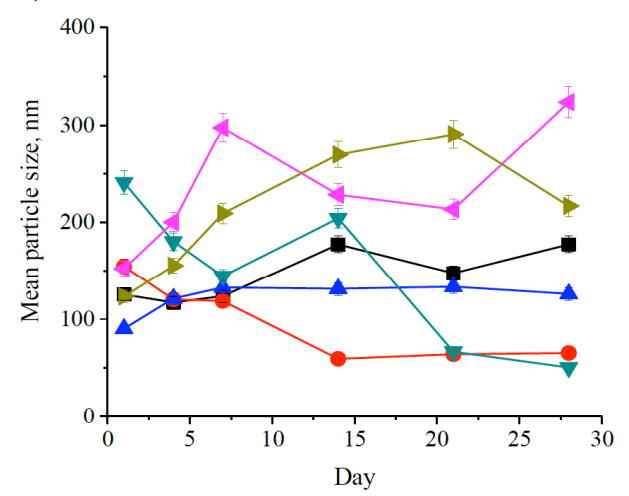

b)

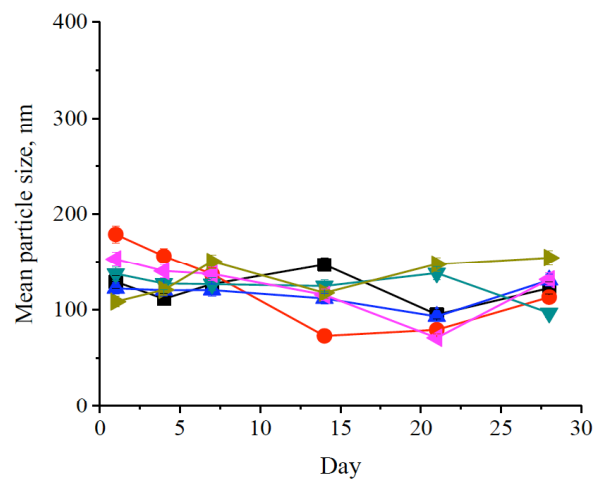

c)

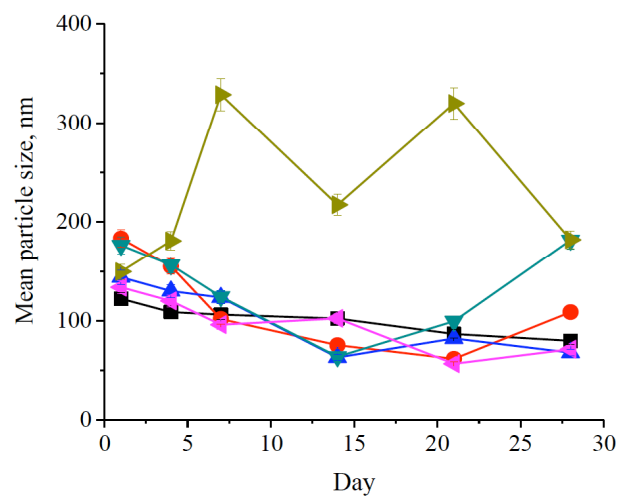

Fig. (2). Mean particle size of C18 fatty acid/DP liposomes: (a) L1/DP, (b) L2/DP, and (c) L3/DP for 28 days at $30^{\circ} \mathrm{C}$. For $\mathbf{m}=$ C18 fatty acids liposomes; the mole ratio of DP to C18 fatty acids: $\bullet=0.01, \boldsymbol{\Delta}=0.02, \boldsymbol{\nabla}=0.03, \boldsymbol{\Lambda}=0.04$, and $\boldsymbol{\nabla}=0.05$ incorporated into liposomes. 
28 days. Their zeta potential was a large negative value of $-45 \mathrm{mV}$ due to their anionic carboxylate head-group. Zeta potential magnitudes of L2 and L3 increased to -25 and $-10 \mathrm{mN} \mathrm{m}^{-1}$ over 14 days of monitoring, respectively (Fig. 3).

(a)

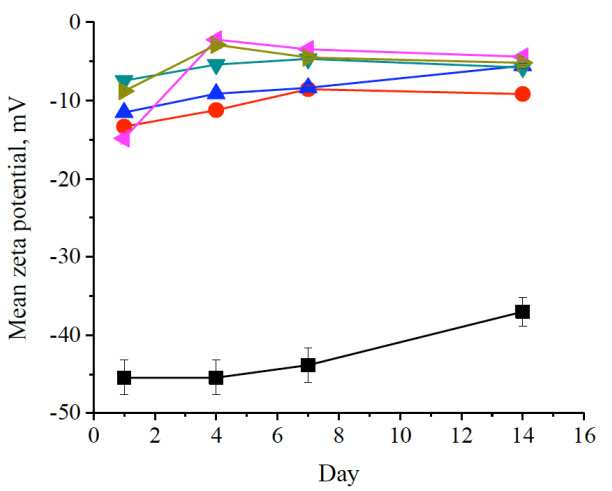

(b)

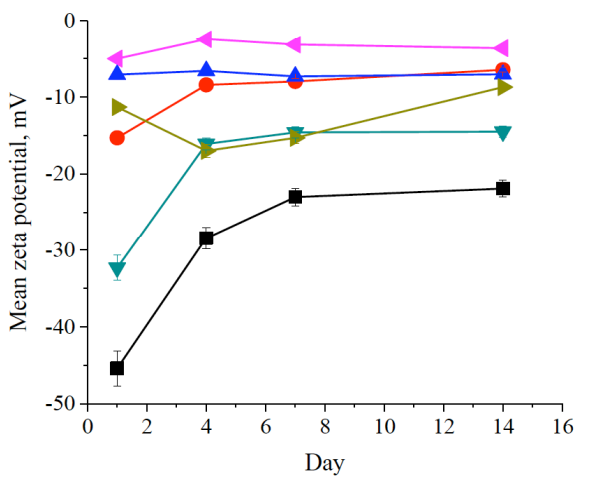

(c)

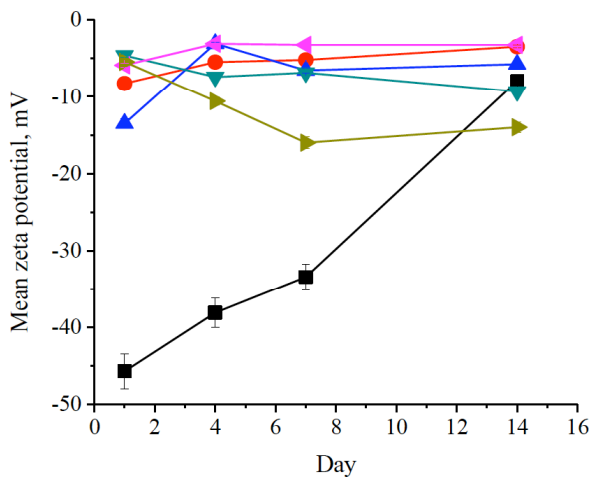

Fig. (3). Mean zeta potential of C18 fatty acid/DP liposomes, (a) $\mathrm{L} 1 / \mathrm{DP}$, (b) $\mathrm{L} 2 / \mathrm{DP}$, and (c) $\mathrm{L} 3 / \mathrm{DP}$ for 14 days at $30^{\circ} \mathrm{C}$. For $\mathbf{\square}=\mathrm{C} 18$ fatty acids liposomes; the mole ratio of DP to C18 fatty acids: $\bullet=0.01, \boldsymbol{\Delta}=0.02, \boldsymbol{\nabla}=0.03, \boldsymbol{\square}=0.04$, and $=0.05$ incorporated into liposomes.

The presence of PEG increases the circulation time up to hours or several days by reducing serum-protein binding. The rate of drug release from nanocarrier, thus, is reduced accordingly. PEG also increases the vascular penetrability of liposomes and facilitates the accumulation of drug-containing liposomes in tumor/cancer tissue [25-29]. The results show that the incorporation of DP into fatty acid liposomes did not entirely improve the particle size and zeta potential stability. The mean particle size measurement for 28 days shows that of L2/DP was more stable than L1/DP and L3/DP (Fig. 2). The mean particle size of L2/DP varied closely within 50 to $200 \mathrm{~nm}$. In contrast, variations in particle size of L1/DP and L3/DP liposomes were observed to fluctuate between 50 and $350 \mathrm{~nm}$. Evident irregularity in particle size was observed in L3/DP at the highest mole ratio of DP (0.05) in the investigation range. The highest mole of DP (in the investigation range) caused the instability of liposomes after 28 days. Zeta potential increased dramatically (Fig. 3) by incorporating DP into pure fatty acid liposomes because DP is less negative-charged than fatty acids that have an overall negative charge. From zeta potential measurements, L1/DP was found to be more stable than L2/DP and L3/DP.

PEGs having saturated lipids such as 1,2 dipalmitoyl-sn-glycero-3-phosphoethanolamine-N[methoxy(polyethylene glycol)-2000] (DPPE PEG2000) [14, 16, 17], and 1,2-distearoyl-snglycero-3-phosphoethanolamine-N-

[amino(polyethylene glycol)-2000] (DSPE PEG2000) $[30,31]$ are commonly used in numerous preparations of stealth liposome (or immunoliposome) formulations for in vivo and in vitro cancer therapeutic studies. PEGs having saturated lipids have neglected bends or kinks in their saturated lipid molecular structures that promote molecular packing, so they are easily inserted into liposome membranes. Fatty acid liposomes incorporated with unsaturated DOPE PEG2000 are slightly larger than liposomes incorporated with saturated DPPE PEG2000 [16, 32]. Such an advantage can be used to encapsulate drugs having large molecule sizes and increase drug encapsulation efficiency of nanocarriers. The unsaturated DOPE hydrocarbon chains of DP increase the repulsion in molecular packing of liposomes due to the effect of cis-double bonds of DOPE, ultimately increasing the size of liposomes.

\subsection{Particle Size and Zeta Potential Analysis of C18 Fatty Acids/AS25 Liposomes}

Irregularity in particle size was found in $\mathrm{C} 18$ fatty acids/AS25 liposomes (Fig. 4). The particle size of L1/AS25 was increased with the increasing 
volume of incorporated AS25 (Fig. 4a). In contrast, L2/AS25 and L3/AS25 did not show evident trend by changing the amount of AS25 (Fig. 4b and $\mathbf{c})$. The particle size of the mixture decreased in less than $100 \mathrm{~nm}$ in 14 days. Presumably, it hydrolyzed into salt solutions during storage. The carboxyl head-group of $\mathrm{C} 18$ fatty acids is rather unstable. PDI of each mixture is slightly larger than that of its respective pure liposome.

(a)

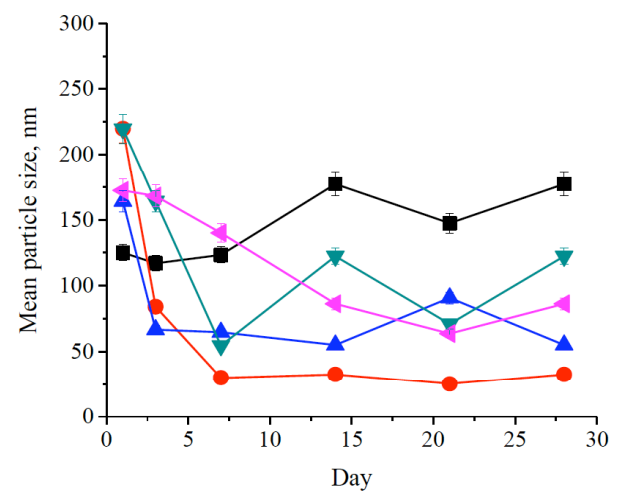

(b)

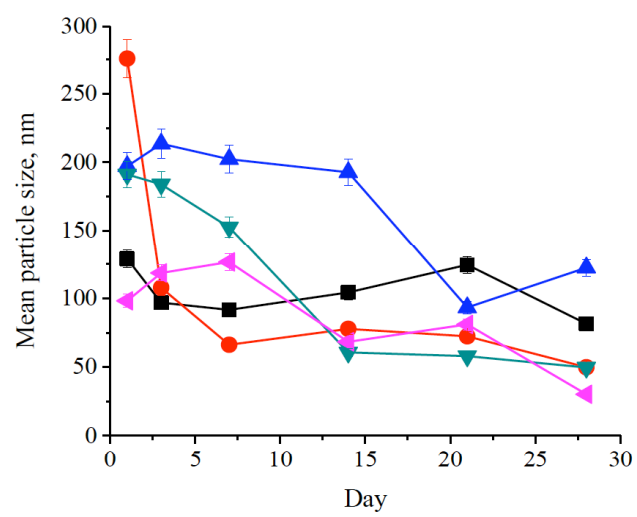

(c)

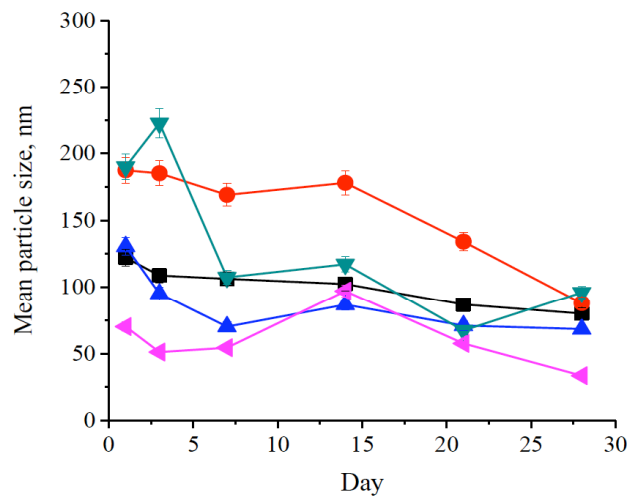

Fig. (4). Mean particle size of C18 fatty acids/AS25 liposomes: (a) L1/AS25, (b) L2/AS25, and (c) L3/AS25 for 28 days at $30^{\circ} \mathrm{C}$. For - = C18 fatty acids liposomes; the amount of AS25: $\bullet=3.36$ nmole, $\boldsymbol{\Delta}=7.40$ nmole, $\boldsymbol{\nabla}=16.8$ nmole, $\boldsymbol{\Psi}=25.2$ nmole incorporated into liposomes.
Charged amino acids present on protein surface can ionize or deionize, depending on the $\mathrm{pH}$, and can make protein a charged particle in aqueous solution. The surface charge of the protein is not static, because the charged groups are acids and bases that constantly exchange protons with water. Therefore, the surface charge of protein is in a dynamic equilibrium with its uncharged state. Because right surface charge on the protein's active site can greatly influence the specific substrate binding, the surface charge of protein is important. Here, zeta potential measurement of AS25 in PBS solution ( $\mathrm{pH} 7.4)$ was found to be $-6.72( \pm 0.15)$. Zeta potential of C18 fatty acid/AS25 liposome solutions were found to be less negative than their highly negative pure liposome solutions. Surface charge of AS25 molecules is less negative than that of C18 fatty acid liposome solutions. Therefore, when AS25 molecules were bounded to the negatively charged surface of $\mathrm{C} 18$ fatty acid liposomes, the surface charge was increased to make them less negative. When $3.36 \mathrm{nmol}$ of AS25 was incorporated into L1 liposome solution, an increase was observed in zeta potential of L1/AS25 from -45 to $-28 \mathrm{mV}$. By increasing the amount of AS25 (8.40 nmol, $16.8 \mathrm{nmol}$, and 25.2 nmol) added into L1 liposome solution, zeta potential also increased and remained between -5 and $-10 \mathrm{mV}$ (Fig. 5a). The zeta potential measured in 14 days also remained at -5 to $-10 \mathrm{mV}$. Zeta potential measurements of L3/AS25 for 14 days showed that the liposome solution was stable and its zeta potential remained between -5 and $-15 \mathrm{mV}$ (Fig. 5c). However, zeta potentials of L1/AS25 and L3/AS25 liposome solutions were more stable than that of L2/AS25 for 14 days, except L2/AS25 containing $25.2 \mathrm{nmol}$ of $\mathrm{AS} 25$, which remained constant at about $-5 \mathrm{mV}$ for 14 days (Fig. 5b).

\subsection{Particle Size and Zeta Potential Analysis of C18 Fatty Acids/DP/AS25 Liposomes}

The combination of active-targeting drug delivery with PEGylation is proven to reduce the rate of drug clearance in blood circulation, modify drug distribution, or even enhance the delivery of therapeutic materials [33-35].

The incorporation of AS25 into PEGylated L1, L2, and L3 liposome solutions stabilized their mean particle size and zeta potential for 28 and 14 days, respectively (Figs. 6 and 7). The mean particle size of L1/DP/AS25 varied from 75 to $125 \mathrm{~nm}$ 
(a)

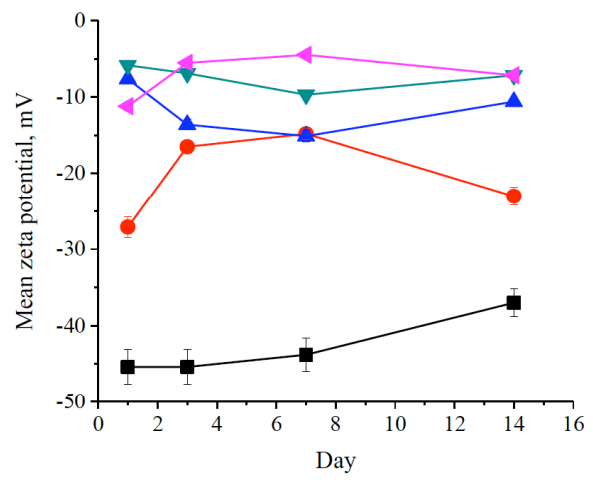

(b)

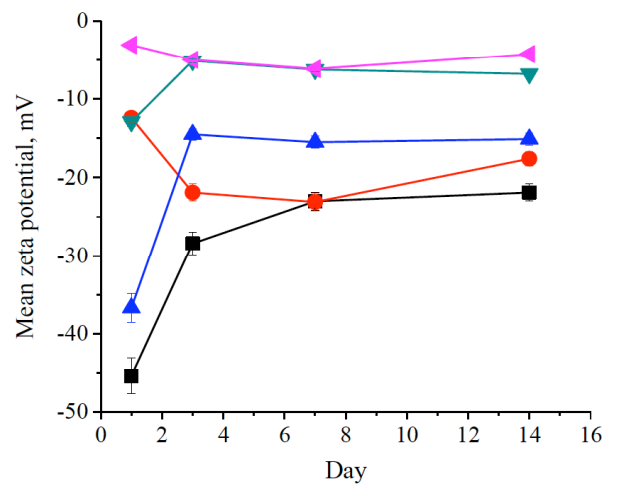

(c)

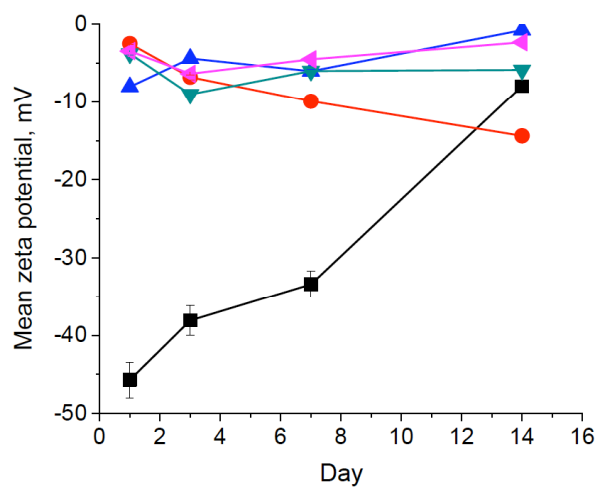

Fig. (5). Mean zeta potential of $\mathrm{C} 18$ fatty acids/AS25 liposomes, (a) L1/AS25, (b) L2/AS25, and (c) L3/AS25 for 14 days at $30^{\circ} \mathrm{C}$. For $=\mathrm{C} 18$ fatty acids liposomes; the amount of AS25: $\bullet=3.36$ nmole, $\boldsymbol{\Lambda}=7.40$ nmole, $\boldsymbol{\nabla}=16.8$ nmole, $\varangle=25.2$ nmole incorporated into liposomes.

and remained constant for 14 days. The zeta potential of L1/DP/AS25 was less negative than that L1/DP having mole ratio 1 to 0.02 , which varied from -12 to $-7.5 \mathrm{mV}$. The surface charge should be more negative when AS25 molecules were bounded to L1/DP surface, because AS25 is more negative than DP. The irregularity of particle size of L2/DP/AS25 was found on day 3 of measurement. The size was reduced to less than $75 \mathrm{~nm}$ on day 7 and increased again to $125 \mathrm{~nm}$ on day 14 . Hydrolysis of L2/DP/AS25 may occur within 7 (a)

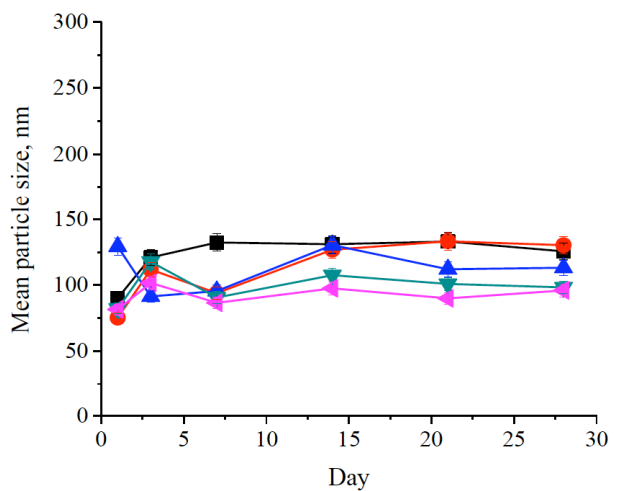

(b)

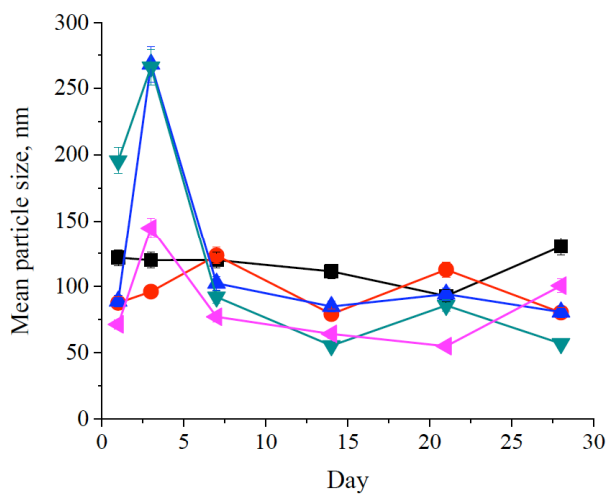

(c)

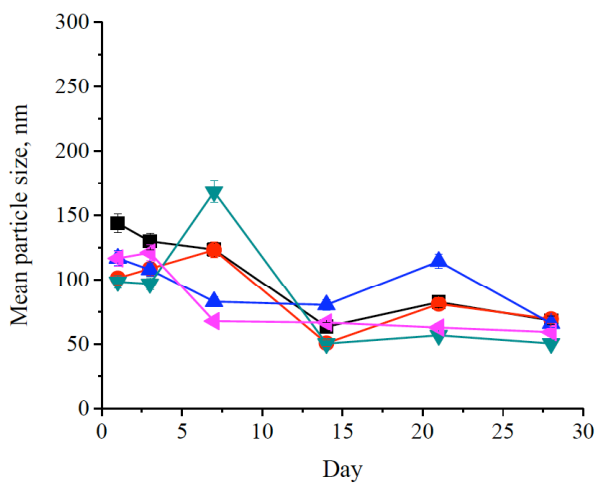

Fig. (6). Mean particle size of $\mathrm{C} 18$ fatty acids/DP/AS25 liposomes: (a) L1/DP/AS25, (b) L2/DP/AS25, and (c) L3/DP/AS25 for 28 days at $30^{\circ} \mathrm{C}$. For $=\mathrm{C} 18$ fatty acids/DP liposomes; the amount of AS25: $\bullet=3.36$ nmole, $\boldsymbol{\Delta}=7.40$ nmole, $\boldsymbol{\nabla}=16.8$ nmole, $\boldsymbol{\Psi}=25.2$ nmole incorporated into liposomes.

days. L2/DP/AS25 might transform into oil droplets on day 14. Zeta potential of L2/DP/AS25 was more negative than that L2/DP having mole ratio of 1 to 0.02 . Presumably, AS25 molecules preferred to be conjugated on L2/DP liposome surface or to the polymer head-group of DP. Mean particle size of L3/DP/AS25 liposome solutions was found to be slightly smaller than that L3/DP having mole ratio of 1 to 0.02 . It varied from 100 to $125 \mathrm{~nm}$, whereas particle size of L3/DP was about $150 \mathrm{~nm}$. In 14 days of particle size monitoring, the particle 
(a)

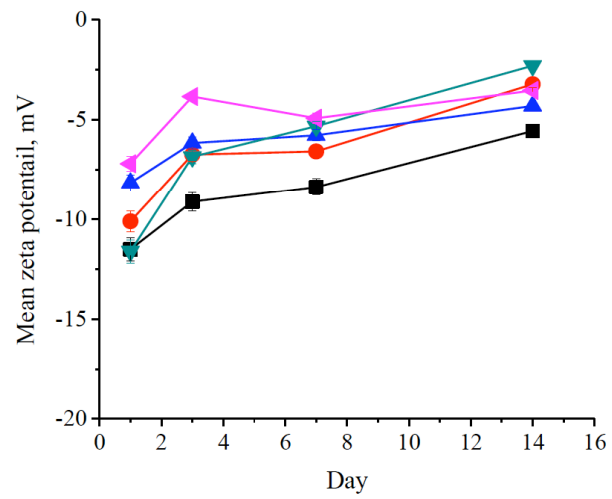

(b)

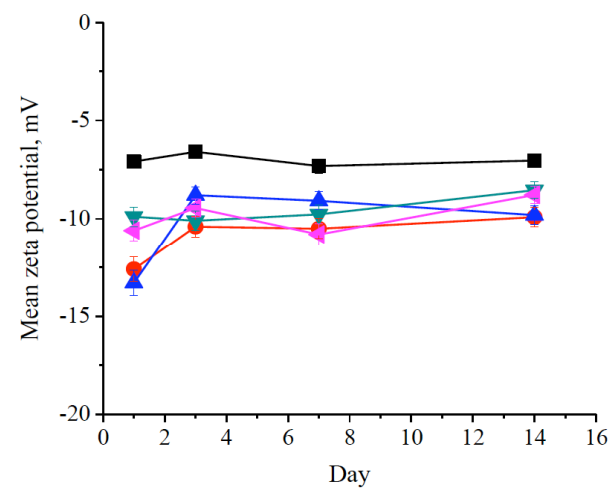

(c)

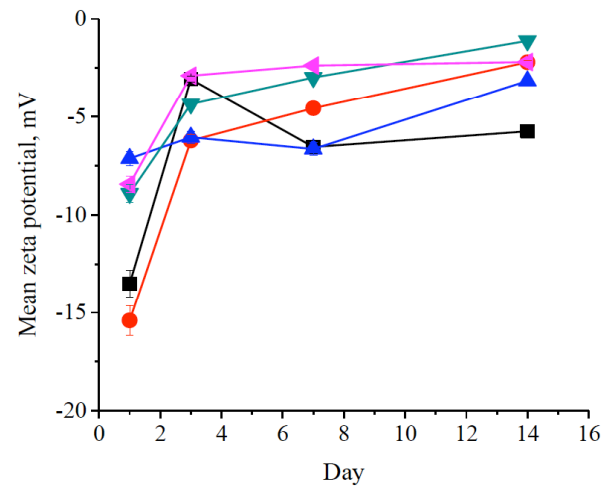

Fig. (7). Mean zeta potential of $\mathrm{C} 18$ fatty acids/DP/AS25 liposomes, (a) L1/DP/AS25, (b) L2/DP/AS25, and (c) $\mathrm{L} 3 / \mathrm{DP} / \mathrm{AS} 25$ for 14 days at $30^{\circ} \mathrm{C}$. For $=\mathrm{C} 18$ fatty acids/DP liposomes; the amount of AS25: $\bullet=3.36$ nmole, $\boldsymbol{\Delta}$ $=7.40$ nmole, $\boldsymbol{\nabla}=16.8$ nmole, $\boldsymbol{\nearrow}=25.2$ nmole incorporated into liposomes.

size of L3/DP reduced to less than $50 \mathrm{~nm}$. This result shows that L3/DP liposome solutions (containing the most number of cis-double bonds) had poor stability and a short shelf life. In addition to the hydrolysis of carboxyl head-group of L3, the cis-double bonds are easily oxidized as well. Precipitations in the vials were observed for L2/DP/AS25 and L3/DP/AS25 liposome solutions, which indicate the instability in correlation to the increasing number of cis-double bonds in hydrocarbon chains of $\mathrm{C} 18$ fatty acids. Zeta potential of
L3/DP/AS25 was less negative than that of L3/DP, except the liposomes solution containing 3.36 nmol of AS25. Zeta potential of liposomes solution containing $3.36 \mathrm{nmol}$ of AS25 was found to be greatly less negative on the third day of monitoring and then remained constant between -2.5 to $-7.5 \mathrm{mV}$ till day 14 for each respective mixture.

The PDI values of PEGylated C18 fatty acids/AS25 liposomes were found to be similar to those of C18 fatty acids/AS25, which varied between 0.40 to 0.55 . The PDI values were larger than PEGylated $\mathrm{C} 18$ fatty acids that had a PDI value between 0.30 to 0.40 . Aggregation would occur when protein molecules were present in liposome solutions.

\subsection{TEM Micrographs}

Figs. (8 and 9) show TEM micrographs of 1 $\mathrm{mM}$ of pure L1, L2, L3, and their PEGylated liposomes, antibody-conjugated $\mathrm{C} 18$ fatty acid liposomes, and PEGylated antibody-conjugated liposomes, respectively. The particle sizes of PEGylated liposomes (L1/DP, L2/DP, and L3/DP) were slightly larger than their pure liposomes (Fig. (a)

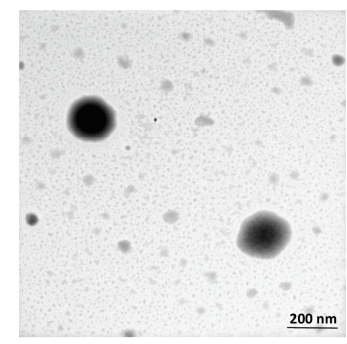

(c)

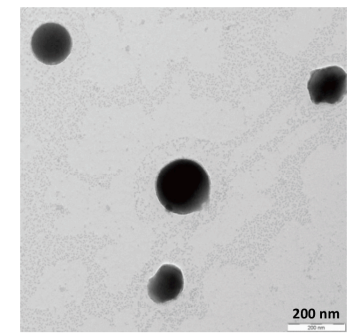

(e)

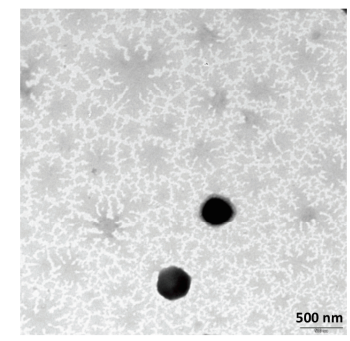

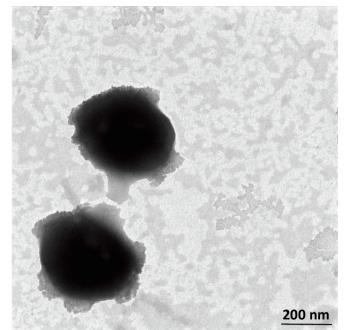

(d)

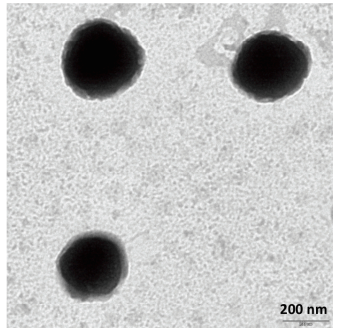

(f)

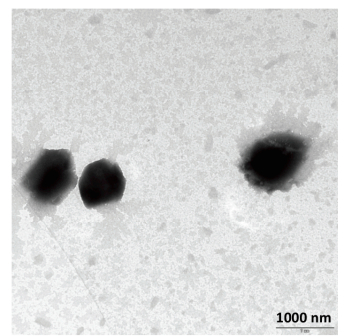

Fig. (8). Transmission electron micrographs of: (a) $1 \mathrm{mM}$ $\mathrm{L} 1$, (b) L1/DP, (c) $1 \mathrm{mM} \mathrm{L} 2$, (d) L2/DP, (e) $1 \mathrm{mM} \mathrm{L3,} \mathrm{(f)}$ L3/DP liposomes at $\mathrm{pH}$ 7.4. 
(a)

(c)
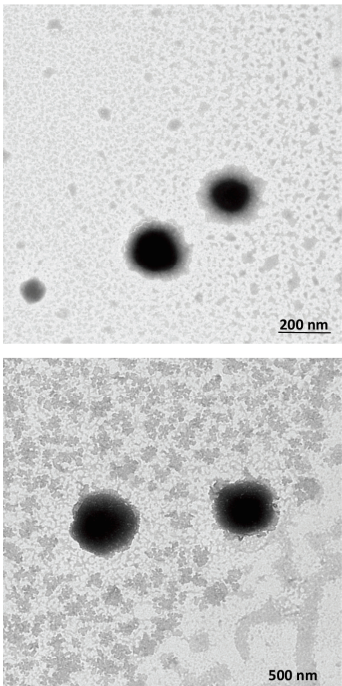

(e)

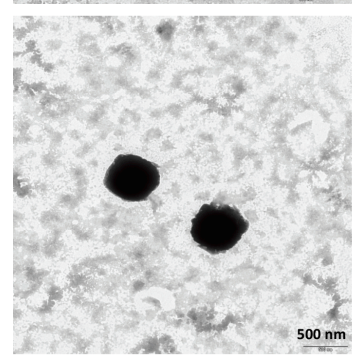

(b)

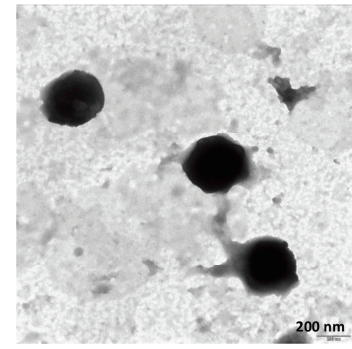

(d)

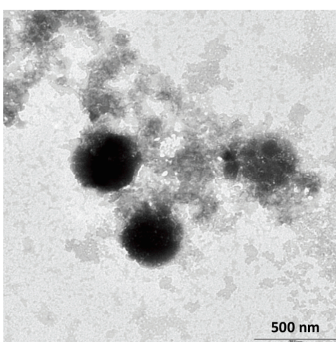

(f)

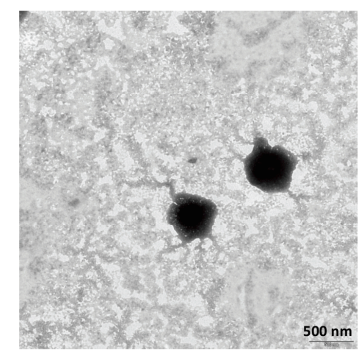

Fig. (9). Transmission electron micrographs of: (a) $1 \mathrm{mM}$ L1/AS25, (b) L1/DP/AS25, (c) $1 \mathrm{mM} \mathrm{L2/AS25,} \mathrm{(d)}$ L2/DP/AS25, (e) $1 \mathrm{mM}$ L3/AS25, (f) L3/DP/AS25 liposomes at $\mathrm{pH} 7.4$.

8). The imperfection of the outer membrane was observed in Fig. (9), which shows the successful incorporation of PEG head-group of DP and AS25 on unsaturated C18 fatty acids liposomes. The particles sizes of liposomes containing AS25 were non-homogenous as shown in Fig. (9), because the sonication was not performed after adding membrane protein AS25 during preparation. The sonication of proteins may cause aggregation and cell lysis.

\section{CONCLUSION}

A rapid development of lipid-based carrier systems is important to overcome the challenges of therapeutic proteins delivery. Membrane-bound protein of AS25 has become the choice of protein to explore lipid-protein interactions. The incorporation of AS25 into PEGylated L1, L2, and L3 liposome solutions stabilized their mean particle size and zeta potentials for 28 and 14 days respectively. The mean particle size of L1/DP/AS25 varied from 75 to $125 \mathrm{~nm}$ and remained constant for 28 days. The zeta potential of L1/DP/AS25, which varied from -12 to $-7.5 \mathrm{mV}$, was less negative than

that L1/DP having the molar ratio of 1 to 0.02 . Therefore, L1/DP/AS25 liposomal system was the most stable PEGylated antibody-targeted liposome system among the studied samples. The stability of C18 fatty acid liposomes was enhanced by incorporating proteins and PEG on the surface as predicted. However, the stability in term of shelf life storage of polyunsaturated fatty acid liposomal formulations remains a challenge. It could be interesting to consider different types of polymers or storage temperatures to stabilize polyunsaturated fatty acid liposomal solutions.

\section{ETHICS APPROVAL AND CONSENT TO PARTICIPATE}

Not applicable.

\section{HUMAN AND ANIMAL RIGHTS}

No Animals/Humans were used for studies that are the basis of this research.

\section{CONSENT FOR PUBLICATION}

Not applicable.

\section{CONFLICT OF INTEREST}

The authors declare no conflict of interest, financial or otherwise.

\section{ACKNOWLEDGEMENTS}

This study was financially supported by the University of Malaya Research Grant Programme SUS (RP022C-16SUS) by University of Malaya, Malaysia. Gew would like to thank Phra Phrom for all blessing.

\section{REFERENCES}

[1] Weiss A, Neuberg P, Philippot S, Erbacher P, Weill CO. Intracellular peptide delivery using amphiphilic lipid-based formulations. Biotech Bioeng 2011; 108(10): 2477-87.

[2] Martins S, Sarmento B, Ferreira DC, Souto EB. Lipid-based colloidal carriers for peptide and protein delivery-liposomes versus lipid nanoparticles. Int $\mathbf{J}$ Nanomed 2007; 2(4): 595.

[3] Pisal DS, Kosloski MP, Balu-Iyer SV. Delivery of therapeutic proteins. J Pharm Sci 2010; 99(6): 255775.

[4] Rawat M, Singh D, Saraf S, Saraf S. Lipid carriers: A versatile delivery vehicle for proteins and peptides. 
Yakugaku zasshi- J Pharm Soc Jap 2008; 128(2): 269-80.

[5] Chang HI, Yeh MK. Clinical development of liposome-based drugs: formulation, characterization, and therapeutic efficacy. Int J Nanomed 2012; 7: 49.

[6] Semple SC, Chonn A, Cullis PR. Interactions of liposomes and lipid-based carrier systems with blood proteins: Relation to clearance behaviour in vivo. Adv Drug Deliv Rev 1998; 32(1-2): 3-17.

[7] Mastrobattista E, Koning GA, Storm G. Immunoliposomes for the targeted delivery of antitumor drugs. Adv Drug Deliv Rev 1999; 40(1-2): 103-27.

[8] Torchilin VP. Recent advances with liposomes as pharmaceutical carriers. Nat Rev Drug Discov 2005; 4(2): 145.

[9] Debotton N, Parnes M, Kadouche J, Benita S. Overcoming the formulation obstacles towards targeted chemotherapy: in vitro and in vivo evaluation of cytotoxic drug loaded immunonanoparticles. J Control Release 2008; 127(3): 219-30.

[10] Laginha K, Mumbengegwi D, Allen T. Liposomes targeted via two different antibodies: assay, B-cell binding and cytotoxicity. Biochim Biophys Acta 2005; 1711(1): 25-32.

[11] Lundberg BB, Griffiths G, Hansen HJ. Cellular association and cytotoxicity of anti-CD74-targeted lipid drug-carriers in B lymphoma cells. J Control Release 2004; 94(1): 155-61.

[12] Park J, Kirpotin D, Hong K, et al. Tumor targeting using anti-her2 immunoliposomes. J Control Release 2001; 74(1-3): 95-113.

[13] Morigaki K, Walde P. Fatty acid vesicles. Curr Opin Colloid Interface Sci 2007; 12(2): 75-80.

[14] Teo YY, Misran M, Low KH, Zain SM. Effect of Unsaturation on the stability of $\mathrm{C} 18$ polyunsaturated fatty acids vesicles suspension in aqueous solution. Bull Korean Chem Soc 2011; 32(1): 59-64.

[15] Tan HW, Misran M. Characterization of fatty acid liposome coated with low-molecular-weight chitosan. J Liposome Res 2012; 22(4): 329-35.

[16] Teo YY, Misran M, Low KH. Effect of $\mathrm{pH}$ on physicochemical properties and encapsulation efficiency of PEGylated linolenic acid vesicles. J Chem 2012; 9(2): 729-38.

[17] Teo YY, Misran M, Low KH. Effect of PEGylated lipid and Lecinol S-10 on physico-chemical properties and encapsulation efficiency of palmitoleate-palmitoleic acid vesicles. J Liposome Res 2014; 24(3): 241-8.

[18] Gew LT, Misran M. Energetic mixing of anti-SNAP25 on lipid monolayers: Degree of saturation of C18 fatty acids. Surf Interf Anal 2017; 49(5): 388-97.

[19] Gew LT, Misran M. Interaction between C18 fatty acids and DOPE PEG2000 in Langmuir monolayers:
Effect of degree of unsaturation. J Biol Phys 2017; 43(3): 397-414.

[20] Hodel A. Molecules in focus: SNAP-25. Int J Biochem Cell Biol 1998; 30: 1069-73.

[21] Rothman JE. The principle of membrane fusion in the cell (Nobel Lecture). Angewandte Chem Int Ed 2014; 53(47): 12676-94.

[22] Torchilin V, Weissig V. Liposomes: A practical approach. US: Oxford University Press 2003.

[23] Samad A, Sultana Y, Aqil M. Liposomal drug delivery systems: An update review. Curr Drug Deliv 2007; 4(4): 297-305.

[24] Résibois-Grégoire A. Electron microscopic studies of metachromatic leucodystrophy. Acta neuropathologica 1967; 9(3): 244-53.

[25] Working P, Newman M, Huang S, Mayhew E, Vaage J, Lasic D. Pharmacokinetics, biodistribution and therapeutic efficacy of doxorubicin encapsulated in Stealth ${ }^{\circledR}$ liposomes (Doxil $\left.{ }^{\circledR}\right)$. J Liposome Res 1994; 4(1): 667-87.

[26] Moghimi SM, Szebeni J. Stealth liposomes and long circulating nanoparticles: Critical issues in pharmacokinetics, opsonization and protein-binding properties. Prog Lipid Res 2003; 42(6): 463-78.

[27] Cattel L, Ceruti M, Dosio F. From conventional to stealth liposomes: A new frontier in cancer chemotherapy. Tumori 2003; 89(3): 237-49.

[28] Immordino ML, Dosio F, Cattel L. Stealth liposomes: Review of the basic science, rationale, and clinical applications, existing and potential. Int $\mathrm{J}$ Nanomed 2006; 1(3): 297.

[29] Nag OK, Awasthi V. Surface engineering of liposomes for stealth behavior. Pharmaceutics 2013; 5(4): 542-69.

[30] Kroon J, Metselaar JM, Storm G, van der Pluijm G. Liposomal nanomedicines in the treatment of prostate cancer. Cancer Treatment Rev 2014; 40(4): 578-84.

[31] Yan F, Li L, Deng Z, et al. Paclitaxel-liposomemicrobubble complexes as ultrasound-triggered therapeutic drug delivery carriers. J Control Release 2013;166(3):246-55.

[32] Suk VRE, Misran M. Development and characterization of DOPE PEG2000 coated oleic acid liposomes encapsulating anticancer drugs. J Surfactants Detergents 2017; 20(2): 321-9.

[33] Luo D, Carter KA, Razi A, et al. Doxorubicin encapsulated in stealth liposomes conferred with light-triggered drug release. Biomaterials 2016; 75: 193-202.

[34] Zappavigna S, Luce A, Porru M, et al. Stealth liposomes for the delivery of zoledronic acid into tumors enhance the anticancer activity of the drug. Translational Med Rep 2017; 1(2): 6596.

[35] Zhang XY, Zhang PY. Polymersomes in nanomedicine - A review. Curr Med Chem 2017; 13(2): 124-9.

DISCLAIMER: The above article has been published in Epub (ahead of print) on the basis of the materials provided by the author. The Editorial Department reserves the right to make minor modifications for further improvement of the manuscript. 